\section{Intestinal apical polarity mediates regulation of TORC1 by glucosylceramide in C. elegans}

Huanhu Zhu, ${ }^{1}$ Aileen K. Sewell, ${ }^{1}$ and Min Han

Howard Hughes Medical Institute, Department of Molecular, Cellular, and Developmental Biology, University of Colorado at Boulder, Boulder, Colorado 80309, USA

TORC1 (target of rapamycin complex 1) plays a central role in regulating growth, development, and behavior in response to nutrient cues. We previously showed that leucine-derived monomethyl branched-chain fatty acids (mmBCFAs) and derived glucosylceramide promote intestinal TORC1 activity for post-embryonic development and foraging behavior in Caenorhabditis elegans. Here we show that clathrin/adaptor protein 1 (AP-1)-dependent intestinal apical membrane polarity and polarity-dependent localization of the vacuolar-type $\mathrm{H}^{+}$-ATPase (V-ATPase) mediate the impact of the lipid pathway on intestinal TORC1 activation. Moreover, NPRL-3 represses mmBCFA-dependent intestinal TORC1 activity at least partly by regulating apical membrane polarity. Our results provide new insights into TORC1 regulation by lipids and membrane polarity in a specific tissue.

Supplemental material is available for this article.

Received April 8, 2015; revised version accepted May 29, 2015.

Regulatory mechanisms that coordinate nutrient availability and metabolic status with growth, development, and behaviors are critical for animals' survival. The TORC1 (target of rapamycin complex 1) pathway is one of the major signaling pathways known to sense the levels of nutrients and metabolites-including amino acids, growth factors, and energy-for a broad range of physiological functions (Zoncu et al. 2011). Many studies indicate that TORC1 is activated by multiple mechanisms to account for variations in upstream signals. For example, the TSC/Rheb and AMPK pathways mediate the growth factor (insulin) and energy level-dependent TORC1 activity, respectively (Laplante and Sabatini 2012; Jewell et al. 2013). Mechanisms of TORC1 activation by certain nutrients such as amino acids and lipids are less clear and appear to be independent of these known signaling pathways (Laplante and Sabatini 2012; Jewell et al. 2013; Zhu et al. 2013). Recent studies have also indicated that different mechanisms are involved in TORC1 activation by different amino acids (Jewell et al. 2015; Wang et al. 2015). Therefore, despite extensive studies

\footnotetext{
[Keywords: Nprl3; Nprl2; mTOR; sphingolipid; VHA-6; branched-chain fatty acid]

${ }^{1}$ These authors contributed equally to this work.

Corresponding author: huanhu.zhu@gmail.com

Article is online at http://www.genesdev.org/cgi/doi/10.1101/gad.263483. 115 .
}

in the TORC1 field, outstanding questions regarding cellular and molecular mechanisms underlying TORC1 activation remain to be answered. In addition, studies using animal models are needed to analyze TORC1 regulation and functions in specific tissues for specific physiological functions.

Caenorhabditis elegans is an excellent model to study TOR signaling-related physiological functions, including development, aging, metabolism and, behaviors /Vellai et al. 2003; Sheaffer et al. 2008; Jones et al. 2009; Soukas et al. 2009; Robida-Stubbs et al. 2012; Webster et al. 2013). We previously established a regulatory connection between a lipid biosynthesis pathway and TORC1 activation in the C. elegans intestine. We showed that monomethyl branched-chain fatty acids (mmBCFAs) and their derived sphingolipid glucosylceramide (GlcCer) impact post-embryonic growth, development, and foraging behavior (Kniazeva et al. 2008, 2015; Zhu et al. 2013). TORC1 activity in the intestine mediates the impact of this lipid pathway, as constitutive TORC1 activity overcomes the physiological defects caused by lipid deficiency (Zhu et al. 2013; Kniazeva et al. 2015). In addition, we showed that the NPRL-2/3 complex negatively regulates this lipid-promoted TORC1 activity (Zhu et al. 2013), consistent with the parallel or subsequent findings in other organisms (Bar-Peled et al. 2013; Panchaud et al. 2013; Wei and Lilly 2014). Here we investigated the mechanism by which these lipids activate intestinal TORC1 activity.

\section{Results and Discussion \\ Identification of factors mediating the regulation of TORC1 by mmBCFA-derived GlcCer}

To identify factors mediating the impact of mmBCFA-derived GlcCer on TORC1 signaling (Fig. 1A), we performed a multiassay, candidate-based RNAi screen (Fig. 1B) based on the following properties shared by both the lipids and TORC1: (1) required for post-embryonic growth and development (loss of function causes larval arrest) (Kniazeva et al. 2004, 2008; Zhu et al. 2013), (2) required for normal foraging behavior (Fig. 1C; Kniazeva et al. 2015), (3) expressed in the intestine (Marza et al. 2009; Seamen et al. 2009; Zhu et al. 2013; Kniazeva et al. 2015), and (4) required for TORC1-dependent nucleolar localization of FIB-1 protein (the C. elegans ortholog of fibrillarin) (Fig. 1D-F; Supplemental Fig. S1B; Sheaffer et al. 2008; Zhu et al. 2013). These properties were further confirmed by RNAi of three other genes known to be involved in the lipid-TORC1 pathway, such as a major TORC1 component (daf-15/raptor) and genes involved in the mmBCFA/sphingolipid de novo pathway (sptl-1 and let-767) (Fig. 1G-I; Supplemental Fig. S1; Entchev et al. 2008; Zhu et al. 2013).

By this approach, we tested 206 candidate genes with the reported larval arrest phenotype (Kamath et al. 2003; Simmer et al. 2003) and found that RNAi knockdown of 120 genes caused a foraging defect (Fig. 1C; Supplemental

(c) 2015 Zhu et al. This article is distributed exclusively by Cold Spring Harbor Laboratory Press for the first six months after the full-issue publication date (see http://genesdev.cshlp.org/site/misc/terms.xhtml). After six months, it is available under a Creative Commons License (Attribution-NonCommercial 4.0 International), as described at http:// creativecommons.org/licenses/by-nc/4.0/. 
A

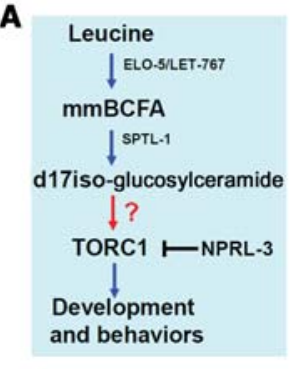

B

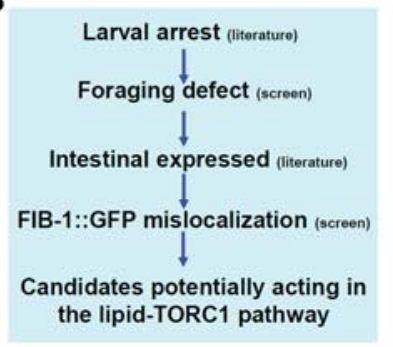

C

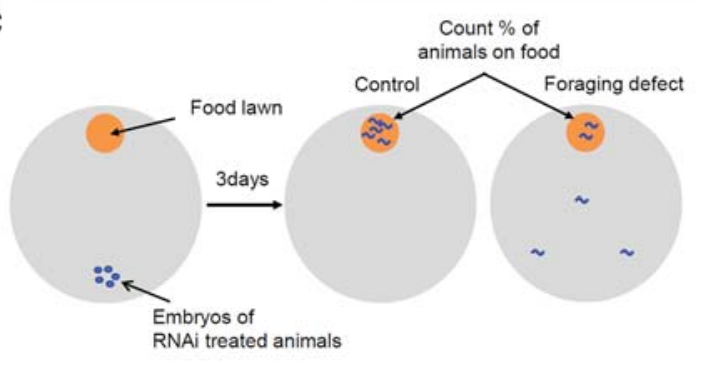

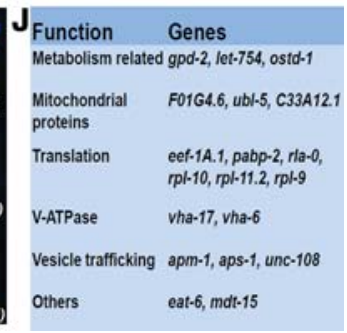

Figure 1. An RNAi screen to identify genes that mediate the impact of mmBCFA-derived GlcCer on intestinal TORC1 activity. (A) A simplified representation of the mmBCFA-GlcCer-TORC1 pathway in $C$. elegans. The red arrow and question mark highlight the step to be probed by the screen. (B) A flow chart showing the screen strategy. All criteria are shared by deficiency of known genes in the lipidTORC1 pathway. $(C)$ A cartoon illustration of the foraging behavior assay used in the screen (see the Materials and Methods; Zhu et al. 2013; Kniazeva et al. 2015 ). (D-I) Representative fluorescence images of the FIB-1::GFP (green) localization assay in intestinal nuclei under various RNAi treatments. In the control condition, FIB-1::GFP showed a condensed nucleolar localization (in the center of the nuclei and with less DAPI signal). In strains treated with RNAi of the indicated genes, FIB-1::GFP was no longer restricted to the nucleoli but showed a punctate or diffuse pattern throughout the nucleus. The quantitative data are presented in Supplemental Figure S1B. (J) A list of the final 19 positive candidate genes from the RNAi screen.

Table 1). Fifty-one of these 120 genes are expressed in the intestine (Materials and Methods; Supplemental Table 2). We then assayed for TORC1 activity by the FIB-1 localization assay and found that RNAi knockdown of 19 genes caused FIB-1 mislocalization (see the Materials and Methods), suggesting that these genes likely have a positive impact on intestine-specific TORC1 activity (Fig. 1J; Supplemental Tables 3, 4).

\section{Lipid-dependent intestinal apical polarity is critical for TORC1 activity}

Among the candidates, two genes (aps-1 and apm-1) encode subunits of the adaptor protein 1 (AP-1) complex (Shim et al. 2000; Boehm and Bonifacino 2001), suggesting a role of AP-1 in the lipid-TORC1 pathway (Fig. 1J; Sup-

plemental Tables 3, 4). Previously, subunits of AP-1, clathrin, and enzymes required for GlcCer synthesis were reported to be essential for intestinal apical polarity maintenance, as loss of function in each of these three pathways resulted in mislocalization of the apical membrane protein ERM-1 (Zhang et al. 2011, 2012). We thus explored whether apical membrane polarity is involved in the impact of mmBCFA-derived lipids on TORC1 function. We first tested the requirement of mmBCFA biosynthesis for intestinal apical membrane polarity and found that blocking mmBCFA biosynthesis by elo-5(RNAi) caused a drastic mislocalization of ERM-1::GFP (Fig. 2A-C; Supplemental Fig. S2) and that the defect was completely suppressed by mmBCFA supplementation (Fig. 2D; Supplemental Fig. S2). These results confirm the hypothesis that the mmBCFA-derived glycosphingolipid (d17isoGlcCer) is required for maintaining apical membrane polarity in the C. elegans intestine.

We then analyzed the functional relationship between intestinal apical membrane polarity and TORC1 activity, since both events require mmBCFAs. We first tested a potential regulatory role of TORC1 on apical polarity and found that RNAi knockdown of two major TORC1 components, let-363/TOR and daf-15/RAPTOR (Hara et al. 2002; Long et al. 2002; Jia et al. 2004), did not disrupt the apical localization of ERM-1::GFP (Fig. 2E,F; Supplemental Fig.
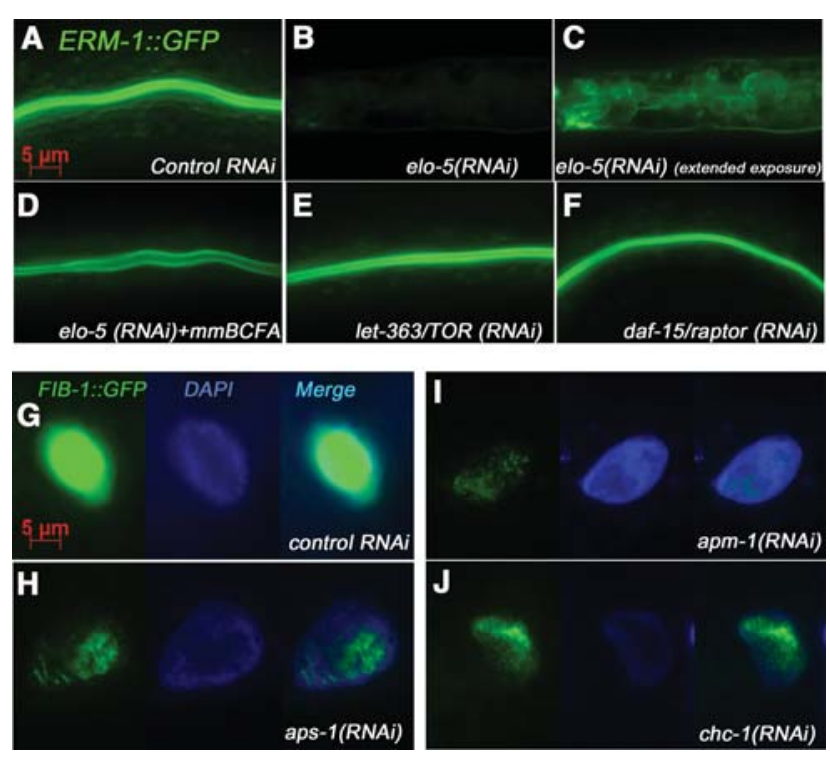

Figure 2. Clathrin/AP-1-dependent intestinal apical membrane polarity is required for the intestinal sphingolipid-TORC1 activity. (A$F$ ) Representative fluorescence images showing ERM-1::GFP (green) expression in the intestine. In the control condition, ERM-1::GFP strictly localizes at the intestinal apical (luminal) membrane. Deficiency of mmBCFA elongase elo-5 caused disorganized localization of ERM-1::GFP in addition to the ERM-1::GFP level decrease (relative GFP intensity: control RNAi $82.8[n=6]$; elo-5 RNAi $14.7[n=17]$; $P=$ $7.6 \times 10^{-7}$ ). The ERM-1::GFP localization was restored by exogenous mmBCFA supplement. RNAi of TORC1 component let-363/TOR or daf-15/raptor did not cause intestinal membrane polarity defects. The statistical data are described in Supplemental Figure S2. $(G-J)$ Representative fluorescence images showing the FIB-1::GFP localization in the intestinal nuclei under the indicated RNAi treatments. Unlike the control, where FIB-1::GFP is condensed in the nucleoli, RNAi of the three genes required for intestinal apical polarity caused a punctate or diffuse pattern of FIB-1::GFP. The statistical data are described in Supplemental Table 3 and Supplemental Figure S1B. 
S2). This indicates that the intestinal polarity defect observed in mmBCFA-deficient animals was not due to a repression of TORC1 activity.

The fact that RNAi targeting AP-1 subunits aps-1 and apm-1 significantly disrupted TORC1-dependent nucleolar localization of FIB-1::GFP (Fig. 2G-I) suggested that AP-1-dependent polarity is potentially required for TORC1 activation. We further investigated this by testing whether disruption of other genes required for apical membrane polarity also down-regulate the intestinal TORC1 activity. In addition to AP-1 components, RNAi knockdown of clathrin component chc-1 (clathrin heavy chain) (Grant and Hirsh 1999) as well as conserved par-6 (partitioning defective protein 6) or dlg-1 (homolog of Drosophila discs large), required for apical polarity (Goldstein and Macara 2007), also mislocalized FIB-1::GFP (Fig. 2J; Supplemental Fig. S1B). In contrast, RNAi of AP-2, which is not required for intestinal apical polarity (Boehm and Bonifacino 2001; Zhang et al. 2012), did not mislocalize FIB-1::GFP (Supplemental Fig. S1B). These data suggest that TORC1 activity is dependent on intestinal apical polarity. This notion is also supported by the data that constitutively active TORC1 suppresses larval arrest caused by mmBCFA deficiency (Zhu et al. 2013), which provides the evidence for TORC1 acting downstream from apical membrane polarity in the intestine. Taken together, these data indicate that the mmBCFA/sphingolipid/clathrin/ AP-1-dependent intestinal apical polarity is critical for TORC1 activity.

mmBCFA-dependent membrane polarity regulates apical localization of the vacuolar-type $H^{+}$-ATPase (V-ATPase) that in turn affects intestinal TORC1 activation

The search described above also identified several subunits, including vha-6, of the V-ATPase, a highly-conserved membrane-bound $\mathrm{H}^{+}$pump (Fig. 1J; Supplemental Tables 1-4; Lee et al. 2010). Further tests of FIB-1::GFP localization showed that multiple V-ATPase subunits are required for intestinal TORC1 activity (Fig. 3A), which is consistent with a previously indicated role of the mammalian V-ATPase in TORC1 activation (Zoncu et al. 2012). Our further analysis also excluded that the vha-6(RNAi) phenotypes are mainly caused by reducing mmBCFA biosynthesis (Supplemental Fig. S3A-F). Because VHA-6 is localized on the intestinal apical membrane (Allman et al. 2009), we hypothesized that its localization may be disrupted by loss of apical membrane polarity in mmBCFAdeficient animals. Indeed, we found that the localization of VHA-6::mCherry at the apical membrane was disrupted; apical-localized VHA-6::mCherry was reduced, and a subset mislocalized to large intestinal vesicles in mmBCFA-deficient worms (Fig. 3B,C; Supplemental Fig. S3G). These defects were completely restored by mmBCFA supplementation (Fig. 3D; Supplemental Fig. S3G). RNAi knockdown of components in the sphingolipid de novo pathway (such as sptl-1) or clathrin/AP-1 pathway (such as chc-1) also caused similar mislocalization defects (Fig. 3F,G; Supplemental Fig. S3G). These defects were not likely to be a secondary result of inactivating TORC1, since VHA-6::mCherry was not mislocalized in animals treated with RNAi of TORC1 components (Fig. $3 E_{\text {; }}$ Supplemental Fig. S3G). These data suggest that the apical localization of VHA-6 significantly contributes to intestinal TORC1 activity.

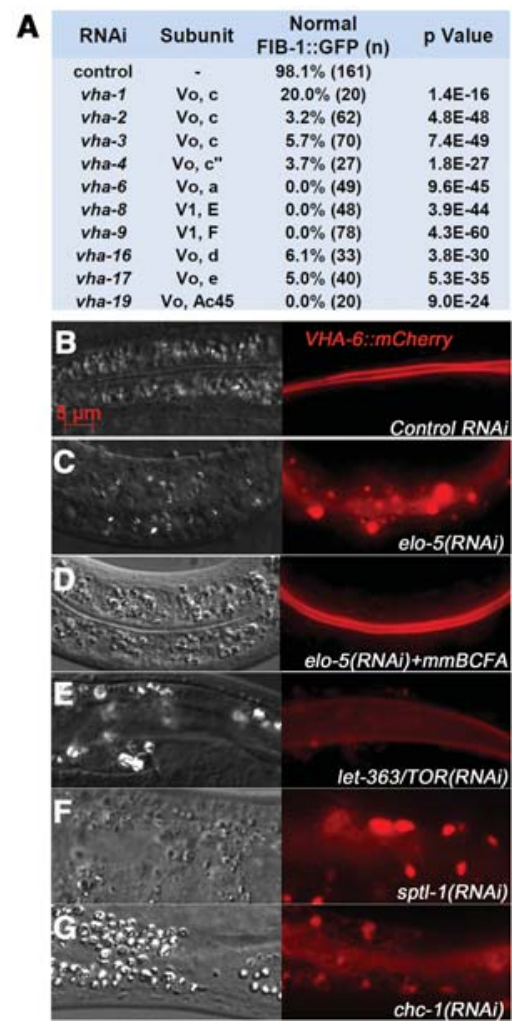

Figure 3. The V-ATPase plays a role in mmBCFA/polarity-mediated TORC1 activation. (A) Statistical data showing that FIB-1::GFP localization in the nucleoli is severely disrupted by RNAi of genes for multiple V-ATPase subunits. $(B-G)$ Fluorescence images showing the expression pattern of VHA- $6:: \mathrm{mCh}$ Crry in the intestine. In the control condition, VHA-6::mCherry was strongly expressed and localized on the intestinal apical membrane. A defect in mmBCFA biosynthesis [elo-5(RNAi)], sphingolipid biosynthesis [sptl-1(RNAi)], or intestinal apical polarity $[$ chc-1(RNAi)], but not in TORC1 $[$ let-363/TOR (RNAi)], caused a diffuse VHA-6::mCherry expression pattern with abnormal accumulation of the signal on large intestinal vesicles. Exogenous mmBCFA fully restored the VHA-6::mCherry mislocalization. The statistical data are described in Supplemental Figure S3G.

\section{NPRL-3 may repress TORC1 activity at least in part by regulating apical membrane polarity}

In our previous work, we identified the NPRL-2/3 complex as the repressor of the mmBCFA-dependent intestinal TORC1 activity in worms (Zhu et al. 2013), which is consistent with the TORC1-repressive roles of this complex identified in parallel studies using mammalian cells and other model organisms (Bar-Peled et al. 2013; Panchaud et al. 2013; Wei and Lilly 2014). Specifically, an nprl-3 loss-of-function (lf) mutation constitutively activates intestinal TORC1 and consequently suppresses the mislocalization of FIB-1 and larval arrest caused by deficiency of mmBCFA or its derived GlcCer (Zhu et al. 2013). Since apical membrane polarity is an upstream event of TORC1 activation in the intestine, as shown above, we asked whether nprl-3(lf) could also restore the intestinal apical polarity in mmBCFA-deficient worms. Such a role would not be expected if NPRL-3 simply represses the function of a key TORC1 component, as was reported in mammalian cells (Bar-Peled et al. 2013). Surprisingly, we found that apical localization of ERM-1:: 
GFP was greatly improved in elo-5(lf) nprl-3(lf) double mutants compared with elo-5(lf) single mutants (Fig. 4A-C; Supplemental Fig. S4A). This nprl-3(lf)-caused polarity restoration is independent of TORC1 activation, since daf-15/raptor RNAi did not disrupt the apical polarity of elo-5(lf) nprl-3(lf) double mutants (Supplemental Fig. S4F-H). In addition, the nprl-3(lf) mutation partially restored the apical localization of VHA-6::mCherry under mmBCFA deficiency (Supplemental Fig. S4B-E). Consistent with these restorations, nprl-3(lf) also partially suppressed the developmental defects caused by the partial loss of function of chc-1, apm-1, and aps-1 (Fig. 4D-H). Combined with the above data showing that TORC1 is not required for apical membrane polarity in the intestine, these results reinforce the conclusion that apical membrane polarity plays a critical role in mediating the impact of the lipid pathway on TORC1 activation in the intestine and suggest that NPRL-3 represses TORC1 activity at least in part by regulating apical membrane polarity.

\section{Perspectives}

Using C. elegans development as a model, we show that clathrin/AP-1-dependent apical membrane polarity, re-
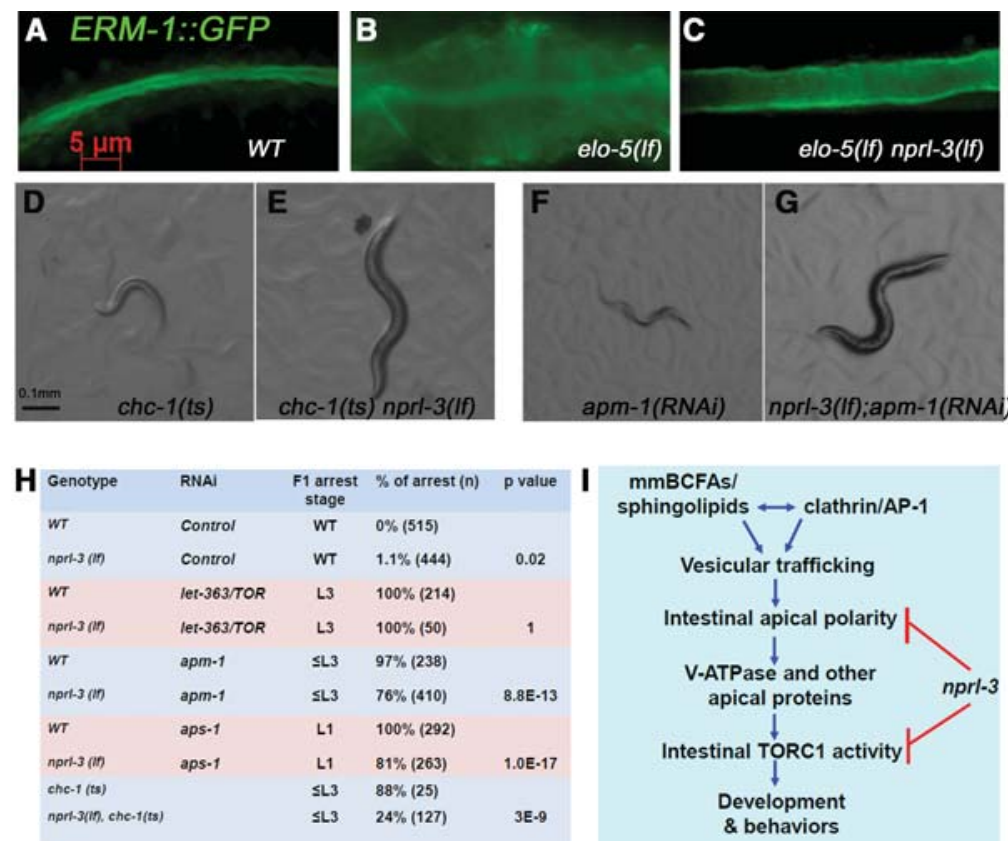

Figure 4. Mutation of nrpl-3 partially rescues the intestinal apical polarity and related defects under mmBCFA deficiency. $(A-C)$ Representative fluorescence images showing ERM-1::GFP expression in the intestine. Disorganized localization of ERM-1::GFP in mmBCFA-deficient animals [elo-5(lf)] was restored by nprl-3(lf). The statistical data are described in Supplemental Figure S4A. $(D-G)$ Representative images showing that nprl-3(lf) partially suppressed the developmental arrest caused by blocking intestinal apical polarity by chc-1(ts) or apm-1(RNAi). (H) Statistical data showing that nprl-3(lf) partially suppressed the developmental arrest caused by blocking intestinal apical polarity [chc-1(ts), apm-1 (RNAi) or aps-1 (RNAi)]. Percentages of larval-arrested animals of the indicated genotypes with the indicated RNA treatment are shown. $P$-values were calculated by the $\chi^{2}$ or Fisher exact test (see the Materials and Methods). (I) A simple model showing the roles of apical polarity, V-ATPase localization, and NPRL-3 in mmBCFA/glycosphingolipid-regulated TORC1 activity in the intestine. The sphingolipid and the clathrin/AP-1 pathway are likely interdependently required for the vesicular trafficking pathway to maintain apical polarity, as previously reported (Zhang et al. 2012). quiring mmBCFA-derived GlcCer, plays a crucial role in TORC1 activation in the intestine (Fig. 4I). Because this

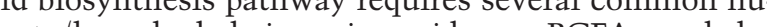
trients (branched-chain amino acids, mmBCFAs, and glucose) and multiple enzymatic reactions (Kniazeva et al. ct al. 2013), the impact of this pathway on mempolarity and TORC1 activity may have important ism pathways affect animal development and behaviors. Although TORC1 activity is a prominent downstream event of apical polarity, the latter is most likely to also afct other cellular signaling events and functions.

Such a role of membrane polarity may not be easily the systems predominantly used to analyze the nutrients. Mechanistic studies of mTORC1 activation by amino acids have focused mainly on specific proteins (such as RagA, V-ATPase, or amino acid transporters) that may mediate the impact of the nutrients on mTORC1 proteins are being regulated under amino acid deprivation is still not clear (Laplante and Sabatini 2012). Whether membrane polarity plays a crucial role in amino acid sensng by TORC1 could be an intriguing question to explore. Another significant finding in this work is that NPRL-3 represses intestinal TORC1 activity at least in part by regulating apical membrane polarity, which is likely the key reason that nprl-3(lf) robustly suppresses the larval developmental defects in mmBCFA-deficient worms (Zhu et al. 2013). This function of the worm NPRL-2/3 complex is distinct from the reported role of the NPRL2/3 complex as a GTPase-activating protein to regulate $\operatorname{RagA} / \mathrm{B}$ in mammalian cell lines and yeast (Bar-Peled et al. 2013; Panchaud et al. 2013). Since our finding was made by analyzing a specific tissue (intestine) for specific physiological roles (development and behavior), it might be important to test the conservation of such a role of the NPRL2/3 complex on membrane polarity using other model organisms.

Our work also supports an essential role for GlcCer to regulate apical membrane polarity and protein localization. Because activating TORC1 and/or restoring the apical polarity suppress most of the developmental/behavioral defects caused by mmBCFA deficiency (Zhu et al. 2013; Kniazeva et al. 2015), maintaining the polarity appears to be an important physiological role of this lipid pathway. Interestingly, mice with enterocyte-specific knockout of the GlcCer synthase gene also showed postnatal lethality with abnormal intestinal structure and nutrient uptake failure in the intestine (Jennemann et al. 2012). The unknown mechanism underlying these defects in mice may be potentially similar or related to the mechanism in $C$. elegans described in this study.

Our data also suggest a role for apical localization of the V-ATPase in TORC1 activation in the intestine, which appears to be distinct from the indicated role of the V-ATPase at the lysosomal membrane in mammalian cells. Although the V-ATPase has been indicated to 
acidify the lysosome lumen in mammalian cells (Lee et al. 2010), it is unlikely that the C. elegans V-ATPase regulates the $\mathrm{pH}$ of lysosome-related organelles (LROs; the worm equivalent of acidified lysosomes in the intestine [Hermann et al. 2005]) because there was no significant difference in LRO $\mathrm{pH}$ between wild-type and elo-5 (RNAi) (Supplemental Fig. S3H-J) or vha-6(RNAi) animals (Allman et al. 2009). This is consistent with the prominent localization of VHA-6::mCherry on the intestinal apical membrane but not on the membranes of LROs (Fig. 3B; Oka et al. 2001). Consistent with the important function of nonlysosomal TORC1 in the worm intestine, TORC1 has also been reported to be activated at nonlysosomal locations in yeast and mammalian tissue culture cells (Betz and Hall 2013). The subcellular localization of TOR in C. elegans has not been reported despite numerous functional studies on the protein, and thus far we have failed to make a functional translational fusion reporter. The exact subcellular location of apical polarity-dependent TORC1 and how the V-ATPase and other proteins on the apical membrane regulate this TORC1 function in the worm intestine remain to be investigated further.

Additional discussion regarding potential mechanisms underlying the regulation of intestinal apical polarity by mmBCFAs and NPRL-3 is presented in the Supplemental Material.

\section{Materials and methods}

\section{Dietary supplements}

Fatty acid C17ISO (Larodan) was prepared as a $10 \mathrm{mM}$ stock in DMSO. A stock solution was mixed with $500 \mu \mathrm{L}$ of OP50 overnight bacterial suspension in a 1:10 ratio and spotted on the appropriate plates (NGM or RNAi) according to the experiment.

\section{Preparation of the mmBCFA-deficient eggs and larvae}

For experiments using elo-5(gk208) mutants, elo-5(gk208) adults maintained on plates with $1 \mathrm{mM}$ C17ISO were washed in M9 buffer and bleached, and eggs were plated on NGM plates spotted with $500 \mu \mathrm{L}$ of OP50 overnight liquid culture. Under these conditions, elo-5(gk208) animals would typically grow to the third larval stage and arrest as mmBCFA-depleted worms.

For experiments using RNAi, adult animals of the corresponding strains were washed off OP50/NGM plates and bleached, and eggs were plated on elo-5(RNAi) plates prepared according to the standard protocol. Adults of the next generation were bleached for eggs, producing C17ISO-deficient L1-arrested larvae.

\section{Lipid analysis by gas chromatography}

The gas chromatography analysis was carried out according to the published method (Kniazeva et al. 2008).

\section{RNAi analysis by feeding}

All RNAi by feeding, except daf-15(RNAi) or let-363(RNAi), used bacterial clones from the Medical Research Council (MRC) RNAi library (Kamath et al. 2003) or the ORF-RNAi library (Open Biosystems). let-363(RNAi) was made as described (Zhu et al. 2013). For the daf-15(RNAi) construct, a PCR fragment of daf-15 genomic DNA (71-871 nucleotides [nt]) was inserted into the L4440 vector by EcoRI/KpnI. For experiments other than the foraging assay, feeding RNAi experiments were done as previously described (Kniazeva et al. 2008). The RNAi-based foraging behavior test method is described in the Supplemental Material.

\section{nprl-3(lf) developmental rescue experiment}

To test developmental rescue of the temperature-sensitive chc-1(b1025) mutant, the $c h c-1$ (b1025) and nprl-3(lf) chc-1(b1025) animals were grown to adults at the permissive temperature $\left(15^{\circ} \mathrm{C}\right)$ and then laid eggs for 2-3 $\mathrm{h}$. To bypass the requirement of $c h c-1$ in the embryonic stage, after removing adults, the eggs were kept at the permissive temperature and then transferred for $24 \mathrm{~h}$ to the restrictive temperature $\left(25^{\circ} \mathrm{C}\right)$ before returning to $15^{\circ} \mathrm{C}$ again. The percentage of animals arrested before the L3 stage were counted. For other experiments, wild-type or nprl-3(lf) adults were bleached on the indicated RNAi plates, were grown to adults, and laid eggs. The percentage of arrested animals was scored in the next generation.

\section{Intestinal apical protein localization test}

The ERM-1::GFP or VHA-6::mCherry transgenic adults were spotbleached on the indicated RNAi bacteria plates. Arrested animals (RNAitreated animals arrested at postnatal day $0[\mathrm{PO}]$ or $\mathrm{F} 1$ generation) or animals at the equivalent stage (RNAi-treated animals without the arrest phenotype) were mounted on a $2 \%$ agar pad with a drop of M9 (plus $25 \mathrm{nM}$ NaN3). Fluorescent images were captured by Nomarski microscopy. The number of animals with normal/abnormal localization was counted.

\section{Statistical analysis}

All statistical analyses (except Lysotracker staining and fluorescence signal quantification experiments) were performed by the $\chi^{2}$ test or Fisher's exact test (when the number of any group in the experiment was smaller than six), and $P<0.05$ was considered a significant difference. Statistical analyses for the Lysotracker staining experiment and fluorescence signal quantification experiments were performed by Student's $t$-test, and $P<0.05$ was considered a significant difference.

Additional Materials and Methods are in the Supplemental Material.

\section{Acknowledgments}

We thank V. Göbel, S. Lo, O. Hobert, K. Sheaffer, S. Mango, S. Takagi, G. Hermann, A. Fire, and the C. elegans Knockout Consortium and Caenorhabditis Genetics Center (funded by the National Institutes of Health [P40OD010440]) for strains and advice. We thank J. Tyler and J. Cavaleri for assistance, and W. Wood, R. Wang, M. Cui, B. Weaver, C. Chi, H. Tang, and our laboratory members for helpful discussions. This work was supported by the Howard Hughes Medical Institute.

\section{References}

Allman E, Johnson D, Nehrke K. 2009. Loss of the apical V-ATPase a-subunit VHA-6 prevents acidification of the intestinal lumen during a rhythmic behavior in C. elegans. Am J Physiol Cell Physiol 297: C1071-C1081.

Bar-Peled L, Chantranupong L, Cherniack AD, Chen WW, Ottina KA Grabiner BC, Spear ED, Carter SL, Meyerson M, Sabatini DM. 2013. A Tumor suppressor complex with GAP activity for the Rag GTPases that signal amino acid sufficiency to mTORC1. Science 340: 11001106.

Betz C, Hall MN. 2013. Where is mTOR and what is it doing there? J Cell Biol 203: 563-574.

Boehm M, Bonifacino JS. 2001. Adaptins: the final recount. Mol Biol Cell 12: 2907-2920.

Entchev EV, Schwudke D, Zagoriy V, Matyash V, Bogdanova A, Habermann B, Zhu L, Shevchenko A, Kurzchalia TV. 2008. LET-767 is required for the production of branched chain and long chain fatty acids in Caenorhabditis elegans. J Biol Chem 283: 17550-17560.

Goldstein B, Macara IG. 2007. The PAR proteins: fundamental players in animal cell polarization. Dev Cell 13: 609-622.

Grant B, Hirsh D. 1999. Receptor-mediated endocytosis in the Caenorhabditis elegans oocyte. Mol Biol Cell 10: 4311-4326.

Hara K, Maruki Y, Long X, Yoshino K, Oshiro N, Hidayat S, Tokunaga C, Avruch J, Yonezawa K. 2002. Raptor, a binding partner of target of rapamycin (TOR), mediates TOR action. Cell 110: 177-189. 
Hermann GJ, Schroeder LK, Hieb CA, Kershner AM, Rabbitts BM, Fonarev P, Grant BD, Priess JR. 2005. Genetic analysis of lysosomal trafficking in Caenorhabditis elegans. Mol Biol Cell 16: 3273-3288.

Jennemann R, Kaden S, Sandhoff R, Nordstrom V, Wang S, Volz M, Robine S, Amen N, Rothermel U, Wiegandt H, et al. 2012. Glycosphingolipids are essential for intestinal endocytic function. I Biol Chem 287: 32598-32616.

Jewell JL, Russell RC, Guan KL. 2013. Amino acid signalling upstream of mTOR. Nat Rev Mol Cell Biol 14: 133-139.

Jewell JL, Kim YC, Russell RC, Yu FX, Park HW, Plouffe SW, Tagliabracci VS, Guan KL. 2015. Metabolism. Differential regulation of mTORC1 by leucine and glutamine. Science 347: 194-198.

Jia K, Chen D, Riddle DL. 2004. The TOR pathway interacts with the insulin signaling pathway to regulate $C$. elegans larval development, metabolism and life span. Development 131: 3897-3906.

Jones KT, Greer ER, Pearce D, Ashrafi K. 2009. Rictor/TORC2 regulates Caenorhabditis elegans fat storage, body size, and development through sgk-1. PLOS Biol 7: e60.

Kamath RS, Fraser AG, Dong Y, Poulin G, Durbin R, Gotta M, Kanapin A, Le Bot N, Moreno S, Sohrmann M, et al. 2003. Systematic functional analysis of the Caenorhabditis elegans genome using RNAi. Nature 421: 231-237.

Kim E, Goraksha-Hicks P, Li L, Neufeld TP, Guan KL. 2008. Regulation of TORCl by Rag GTPases in nutrient response. Nat Cell Biol 10: 935-945.

Kniazeva M, Crawford QT, Seiber M, Wang CY, Han M. 2004. Monomethyl branched-chain fatty acids play an essential role in Caenorhabditis elegans development. PLoS Biol 2: E257.

Kniazeva M, Euler T, Han M. 2008. A branched-chain fatty acid is involved in post-embryonic growth control in parallel to the insulin receptor pathway and its biosynthesis is feedback-regulated in C. elegans. Genes Dev 22: 2102-2110.

Kniazeva M, Zhu H, Sewell AK, Han M. 2015. A lipid-TORC1 pathway promotes neuronal development and foraging behavior under both fed and fasted conditions in C. elegans. Dev Cell 33: 260-271.

Laplante M, Sabatini DM. 2012. mTOR signaling in growth control and disease. Cell 149: 274-293.

Lee SK, Li W, Ryu SE, Rhim T, Ahnn J. 2010. Vacuolar (H+)-ATPases in Caenorhabditis elegans: what can we learn about giant $\mathrm{H}+$ pumps from tiny worms? Biochim Biophys Acta 1797: 1687-1695.

Long X, Spycher C, Han ZS, Rose AM, Muller F, Avruch J. 2002. TOR deficiency in C. elegans causes developmental arrest and intestinal atrophy by inhibition of mRNA translation. Curr Biol 12: $1448-1461$.

Marza E, Simonsen KT, Faergeman NJ, Lesa GM. 2009. Expression of ceramide glucosyltransferases, which are essential for glycosphingolipid synthesis, is only required in a small subset of C. elegans cells. J Cell Sci 122: 822-833.

Oka T, Toyomura T, Honjo K, Wada Y, Futai M. 2001. Four subunit a isoforms of Caenorhabditis elegans vacuolar $\mathrm{H}^{+}$-ATPase. Cell-specific expression during development. J Biol Chem 276: 33079-33085.
Panchaud N, Peli-Gulli MP, De Virgilio C. 2013. Amino acid deprivation inhibits TORC1 through a GTPase-activating protein complex for the Rag family GTPase Gtr1. Sci Signal 6: ra42.

Robida-Stubbs S, Glover-Cutter K, Lamming DW, Mizunuma M, Narasimhan SD, Neumann-Haefelin E, Sabatini DM, Blackwell TK. 2012. TOR signaling and rapamycin influence longevity by regulating SKN-1/Nrf and DAF-16/FoxO. Cell Metab 15: 713-724.

Seamen E, Blanchette JM, Han M. 2009. P-type ATPase TAT-2 negatively regulates monomethyl branched-chain fatty acid mediated function in post-embryonic growth and development in C. elegans. PLoS Genet 5: e1000589.

Sheaffer KL, Updike DL, Mango SE. 2008. The target of rapamycin pathway antagonizes pha-4/FoxA to control development and aging. Curr Biol 18: 1355-1364.

Shim J, Sternberg PW, Lee J. 2000. Distinct and redundant functions of mul medium chains of the AP-1 clathrin-associated protein complex in the nematode Caenorhabditis elegans. Mol Biol Cell 11:2743-2756.

Simmer F, Moorman C, van der Linden AM, Kuijk E, van den Berghe PV, Kamath RS, Fraser AG, Ahringer J, Plasterk RH. 2003. Genome-wide RNAi of C. elegans using the hypersensitive rrf-3 strain reveals novel gene functions. PLOS Biol 1: E12.

Soukas AA, Kane EA, Carr CE, Melo JA, Ruvkun G. 2009. Rictor/TORC2 regulates fat metabolism, feeding, growth, and life span in Caenorhabditis elegans. Genes Dev 23: 496-511.

Vellai T, Takacs-Vellai K, Zhang Y, Kovacs AL, Orosz L, Muller F. 2003. Genetics: influence of TOR kinase on lifespan in C. elegans. Nature 426: 620 .

Wang S, Tsun ZY, Wolfson RL, Shen K, Wyant GA, Plovanich ME, Yuan ED, Jones TD, Chantranupong L, Comb W, et al. 2015. Metabolism. Lysosomal amino acid transporter SLC38A9 signals arginine sufficiency to mTORC1. Science 347: 188-194.

Webster CM, Wu L, Douglas D, Soukas AA. 2013. A non-canonical role for the C. elegans dosage compensation complex in growth and metabolic regulation downstream of TOR complex 2. Development 140:3601-3612.

Wei Y, Lilly MA. 2014. The TORC1 inhibitors Nprl2 and Nprl3 mediate an adaptive response to amino-acid starvation in Drosophila. Cell Death Differ 21: 1460-1468.

Zhang H, Abraham N, Khan LA, Hall DH, Fleming JT, Gobel V. 2011. Apicobasal domain identities of expanding tubular membranes depend on glycosphingolipid biosynthesis. Nat Cell Biol 13: 1189-1201.

Zhang H, Kim A, Abraham N, Khan LA, Hall DH, Fleming JT, Gobel V. 2012. Clathrin and AP-1 regulate apical polarity and lumen formation during C. elegans tubulogenesis. Development 139: 2071-2083.

Zhu H, Shen H, Sewell AK, Kniazeva M, Han M. 2013. A novel sphingolipid-TORC1 pathway critically promotes postembryonic development in Caenorhabditis elegans. Elife 2: e00429.

Zoncu R, Efeyan A, Sabatini DM. 2011. mTOR: from growth signal integration to cancer, diabetes and ageing. Nat Rev Mol Cell Biol 12: 21-35.

Zoncu R, Bar-Peled L, Efeyan A, Wang S, Sancak Y, Sabatini DM. 2012. mTORC1 senses lysosomal amino acids through an inside-out mechanism that requires the vacuolar $\mathrm{H}^{+}$-ATPase. Science 334: 678-683. 


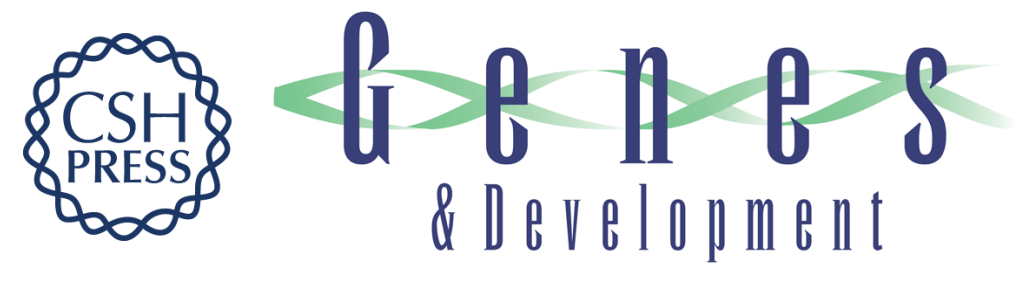

\section{Intestinal apical polarity mediates regulation of TORC1 by glucosylceramide in $C$. elegans}

Huanhu Zhu, Aileen K. Sewell and Min Han

Genes Dev. 2015, 29:

Access the most recent version at doi:10.1101/gad.263483.115

\section{Supplemental Material \\ http://genesdev.cshlp.org/content/suppl/2015/06/24/29.12.1218.DC1}

References

This article cites 40 articles, 18 of which can be accessed free at: http://genesdev.cshlp.org/content/29/12/1218.full.html\#ref-list-1

Creative

Commons

License

Email Alerting

Service

This article is distributed exclusively by Cold Spring Harbor Laboratory Press for the first six months after the full-issue publication date (see

http://genesdev.cshlp.org/site/misc/terms.xhtml). After six months, it is available under a Creative Commons License (Attribution-NonCommercial 4.0 International), as described at http://creativecommons.org/licenses/by-nc/4.0/.

Receive free email alerts when new articles cite this article - sign up in the box at the top right corner of the article or click here.

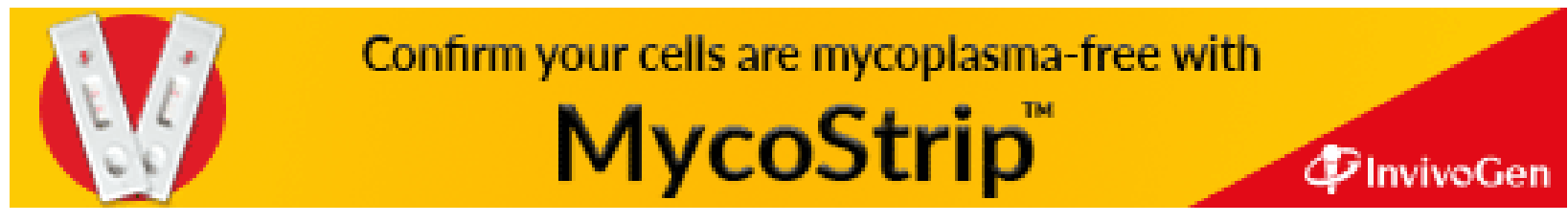

